\title{
Expendable bubble tiltmeter for geophysical monitoring
}

\author{
J. A. Westphal, M. A. Carr, and W. F. Miller ${ }^{a)}$ \\ Division of Geological and Planetary Sciences, California Institute of Technology, Pasadena, California 91125
}

Daniel Dzurisin

\author{
U.S. Geological Survey, David A. Johnston Cascades Volcano Observatory, 5400 MacArthur Blvd., \\ Vancouver, Washington 98661
}

(Received 13 October 1982; accepted for publication 21 December 1982)

\begin{abstract}
An unusually rugged highly sensitive and inexpensive bubble tiltmeter has been designed, tested, and built in quantity. These tiltmeters are presently used on two volcanoes and an Alaskan glacier, where they continuously monitor surface tilts of geological interest. This paper discusses the mechanical, thermal, and electric details of the meter, and illustrates its performance characteristics in both large $\left(>10^{-4}\right.$ radian $)$ and small $\left(<10^{-6}\right.$ radian $)$ tilt environments. The meter's ultimate sensitivity is better than $2 \times 10^{-8}$ radians rms for short periods (hours), and its useful dynamic range is greater than $10^{4}$. Included is a short description of field use of the instrument for volcano monitoring.
\end{abstract}

PACS numbers: $91.30 .-\mathrm{f}, 93.85 .+\mathrm{q}$

\section{INTRODUCTION}

Tiltmeters of various designs have long been used to monitor and study active volcanoes. ${ }^{1}$ Observed tilt changes range from less than ten to thousands of $\mu \mathrm{rad}\left(1 \mu \mathrm{rad}=10^{-6} \mathrm{radi}-\right.$ an), with geologically interesting changes on time scales of minutes to years. Used together, continuous tilt measurements and seismic monitoring provide an effective tool for short-term prediction of eruptions. ${ }^{2}$ Although some volcanoes are sufficiently accessible and benign to allow installation and maintenance of permanent tiltmeter stations (cf. Kilauea and Mauna Loa, Hawaii), many are too remote for frequent visits or too hostile for expensive instrumentation.

The catastrophic eruption of Mount St. Helens in May $1980^{3}$ triggered broad interest in geophysical measurements that might help predict future events, and determine physical conditions on and within the volcano. The tiltmeter described in this paper was designed specifically for use in the crater at Mount St. Helens. Although it is intended to be expandable (i.e., cheap), it has well-behaved reproducible characteristics, uses a minimum of electrical power, and resists severe environmental conditions. It is both light and compact and can be installed quickly with only hand tools. Since a major motive for automated tilt measurements at Mount St. Helens is to aid in eruption prediction, the tiltmeter was designed to be compatible with a USGS digital telemetry system which transmits data $75 \mathrm{~km}$ from the volcano to the Cascades Volcano Observatory in Vancouver, Washington.

\section{THE SENSOR}

Electrolytic bubble tilt sensors were, to our knowledge, first described in the literature by Cooper, ${ }^{4}$ who used a biaxial bubble to remotely level inertial guidance systems. Commercially available tiltmeters using this Rockwell transducer $^{5}$ have been used for monitoring potential earthquake producing faults in California and elsewhere, ${ }^{6-8}$ and for monitoring active volcanoes in Hawaii. ${ }^{9}$
Unfortunately, the Rockwell sensor is relatively expensive and not conveniently available. However, a single axis bubble sensor with about the same sensitivity and noise characteristics as the Rockwell sensor is now commercially available at considerably lower cost. Two of these Fredericks ${ }^{10,11}$ sensors can be mounted orthogonally to provide biaxial tilt data.

Figure 1 is a schematic diagram of the Fredericks sensor. A 30-mm-long glass tube of about $8-\mathrm{mm}$ o.d. and about 6-mm bore is bent to a radius of $355 \mathrm{~mm}$. Three wires $\left(\mathrm{A}, \mathrm{A}^{\prime}\right.$, and $B$ ) are sealed into the tube to form three electrodes. The tube is partially filled with an electrically conducting fluid entirely covering wire $B$, but leaving a bubble into which wires $A$ and $A^{\prime}$ penetrate. The nature of the wires, electrolyte, and gas in the bubble, as well as any special preparations of the glass bore, are proprietary information of the manufacturer.

When the sensor is tilted counterclockwise as seen in Fig. 1, the bubble moves to the position shown by the dotted lines, covering more of wire $A$ and uncovering more of $A^{\prime}$. Movement of the bubble caused by tilting is sensed by measuring the change in conductivity of the fluid path between wires $A$ and $B$ and between $A^{\prime}$ and $B$. Since the fluid is an electrolyte, the conductivity is measured with alternating

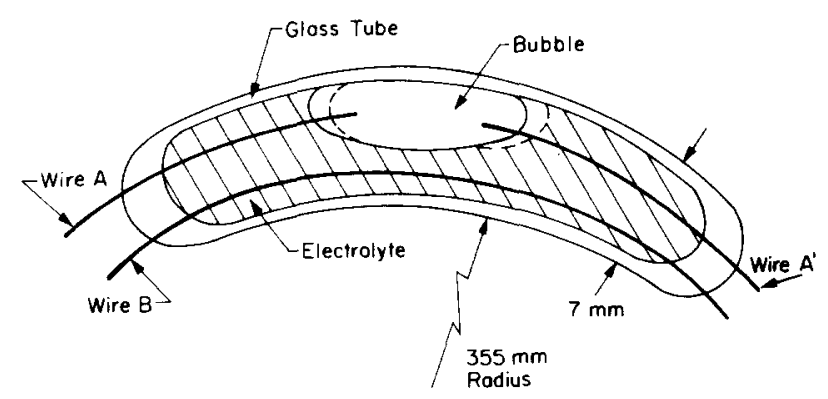

FIG. 1. Cross-section schematic diagram of the Fredericks one-axis bubble tilt sensor. 
current to prevent electrolysis and the formation of hydrogen and oxygen gases, which might change the size of the bubble.

\section{ELECTRONICS}

Figure 2 shows a block diagram of the electronic circuits used to sense changes in fluid path conductivity in the Fredericks sensor. The bubble is arranged as two legs of an ac Wheatstone bridge, with fixed resistors in the other two legs. The bridge output is then differentially amplified and synchronously detected to give a dc output, which is lowpass filtered with a time constant of about $100 \mathrm{~s}$ to remove the effects of natural $8 \mathrm{~s}$ "microseisms" (indeed this type of sensor may also be used as a seismometer"

Because even very small dc currents through the sensor may cause long term bubble growth and resulting drift, the bridge square-wave drive and the input amplifier are transformer coupled. Each leg of the sensor has an impedance of about $1000 \Omega$, and the fixed resistors are each $1000 \Omega$.

With normal construction techniques the bridge is not purely resistive, and with a $1-\mathrm{kHz}$ square-wave drive any capacitive unbalance of the bridge is important. Although it is possible to exactly balance the bridge by the use of trim capacitors under laboratory conditions, the thermal and mechanical environment in normal field use is so variable as to require another means of removing residual variable capacitive effects. By using a square-wave drive, these effects can be isolated to about the first $50 \mu$ s after each square-wave transition. A phase locked analog gate may then "chop" out the first $50 \mu \mathrm{s}$ of each signal transition, before it is fed to the synchronous detector, thus removing any effect of variable amplitude due to capacitive changes.

All other features of the electronics are conventional. To minimize power consumption, CMOS and low-power operational amplifiers are used throughout. Table I shows the transfer functions ${ }^{4}$ for both the Fredericks and Rockwell sensors.

\section{THERMAL AND MECHANICAL CONSIDERATIONS}

Because portability and easy installation are crucial at dangerous and remote field sites, it is desirable that geophysical tiltmeters be compact. The exceedingly small angles and dimensions involved in these tiltmeters require careful mechanical design. Recognizing that $1 \mu \mathrm{rad}$ means a slope of $1 \mu / \mathrm{m}$, it is easy to see that thermal gradients must be minimized and that all mechanical interfaces must be

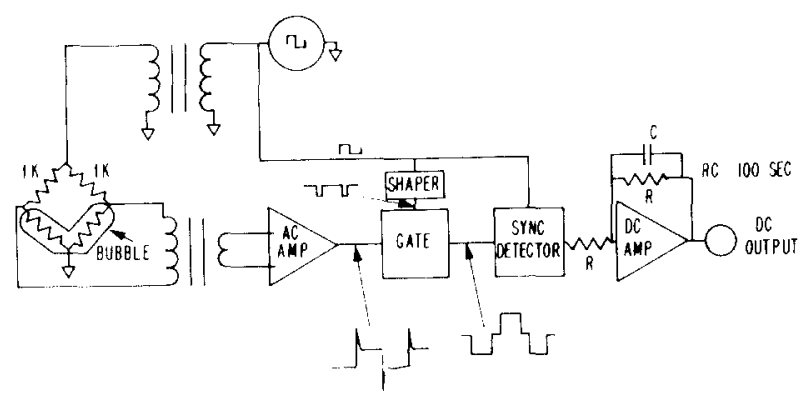

FIG. 2. Block diagram of the tiltmeter electronics.
TABLE I. Transfer functions of the Rockwell and Fredericks bubble sensors.

Transfer function: Tilt and acceleration to volts

$$
E_{B(s)}=\left(K_{p}\left[\left(s^{2}-\omega_{0}^{2}\right) \phi_{(s)}+(1 / R) s^{2} X_{(s)}\right)\right] /\left(s^{2}+25 \omega_{0}+\omega_{0}^{2}\right)
$$

$R \quad$ is the radius of curvature of the lid or tube where the electrolyte bubble forms

$\omega_{0} \quad$ is the natural angular resonant frequency

$K_{p} \quad$ is the pick-off scale factor of the bridge used to detect the position of the bubble

$\zeta \quad$ is the coefficient of damping

$E_{B|s|} \quad$ is the Laplace transform of the output voltage of the sensor and its bridge circuit

$s \quad$ is the complex variable of the Laplace transformation

$X_{|s|} \quad$ is the Laplace transform of the bubble displacement

$\phi_{|,| s \mid} \quad$ is the Laplace transform of the tilt of the sensor about the center of curvature of the bubble lid or tube

\begin{tabular}{lll} 
& Rockwell & Fredericks \\
$R$ & 0.305 & $0.356 \mathrm{~m}^{\mathrm{a}}$ \\
$\omega_{\mathrm{D}}^{\mathrm{d}}$ & 5.67 & $5.25 \mathrm{rad} / \mathrm{s}$ \\
& $\left(\tau_{0}=1.1 \mathrm{~s}\right)$ & $\left(\tau_{0}=1.2 \mathrm{~s}\right)$ \\
$K_{p}$ & 42.6 & $27.7 \mathrm{Vrms} / \mathrm{rad}^{\mathrm{b}}$ \\
$\zeta$ & 1.0 & $3.7^{\circ}$ \\
$\begin{array}{l}\text { Electrolytic } \\
\text { impedance }\end{array}$ & $8000 \Omega^{\mathrm{c}}$ & $1000 \Omega^{\mathrm{e}}$ \\
$\begin{array}{l}\text { Overall scale } \\
\text { factor }\end{array}$ & 40 & $49 \mathrm{mV} / \mu \mathrm{rad}^{\mathrm{d}}$ \\
\hline
\end{tabular}

The natural period was computed from $\omega_{0}=(g / R)^{1 / 2}$.

${ }^{b}$ This value is normalized to a $1-V$ rms bridge excitation and bridge resistors equal to the nominal bubble resistance. The $K_{p}$ given is the average of five static tilt tests on two Fredericks bubbles. Accuracy is $\approx \pm 15 \%$. The normalized value based upon the Fredericks specification should be 61.9 Vrms/rad.

"This value was determined from six dynamic tilt tests on two Fredericks bubbles by making a least-squares fit of the signal decay vs time to the exponential equation $y=a e^{-t} / \tau$. The average value of $\tau$ was $8.6 \mathrm{~s}$. By knowing this dominant pole, the other pole and the coefficient of damping, $\xi$, were determined.

d $49 \mathrm{mV} / \mu \mathrm{rad}$ is the output scale factor when using the Caltech Rockwell bubble electronics.

'From the manufacturer's specification data.

very stable if a tiltmeter is to operate reliably with $\mu \mathrm{rad}$ sensitivity.

Figure 3 shows, schematically, the mechanical mounting of the Fredericks sensors used for volcano monitoring. The meter is placed on a smooth ceramic floor tile which has been cemented to as solid a surface as is available at the field

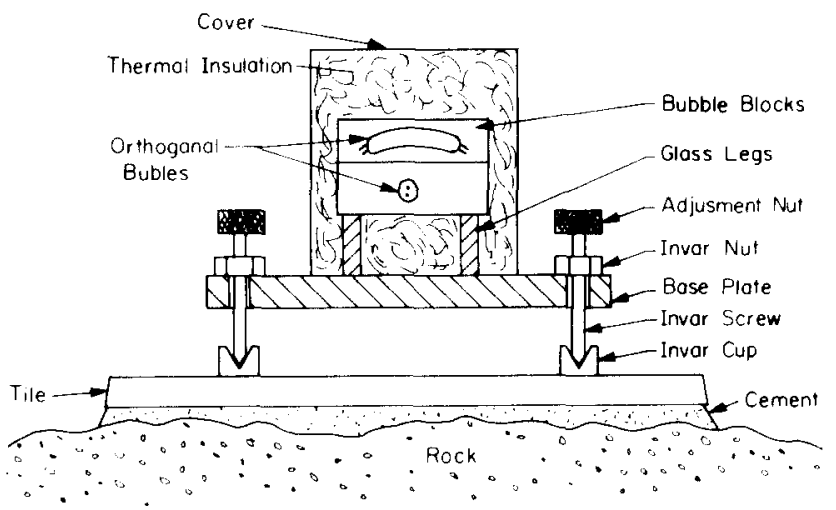

FiG. 3. A cut-away view of the tiltmeter used for volcano monitoring. 
site (the details of our experience in forming geophysically acceptable couplings to the earth is outside the scope of this paper). Three leveling screws attached to the instrument baseplate rest in conical holes in small metal cups, which are bonded to the tile surface to prevent lateral movement of the meter during earthquakes. The two sensors are orthogonally mounted in metal blocks which are attached to the baseplate with glass legs, and are surrounded by glass fiber insulation encased in a metal cover. A temperature sensor is mounted in the baseplate and, with the tilt sensors, is connected electrically to the electronics unit by a cable up to $10 \mathrm{~m}$ long.

There are a number of special features in this design. Since the tile cannot be installed exactly level, the meter must be accurately leveled by changing the length of the adjusting screws which are about $100 \mathrm{~mm}$ apart. Because the screws do not have a zero thermal expansion coefficient, differences in their lengths cause the baseplate to tilt when the temperature changes. Even with Invar, which has the lowest coefficient of thermal expansion of any convenient material, this effect is significant. The temperature induced tilt is given by

$$
\phi=K \Delta L / D,
$$

where $\phi$ is the tilt in radians $/{ }^{\circ} \mathrm{C} ; \Delta L$ is the difference in screw length; $K$ is the linear thermal expansion coefficient per degree Celsius, and $D$ is the horizontal distance between screws. For our design $K=1.25 \times 10^{-6} /{ }^{\circ} \mathrm{C}$ and $D=100$ $\mathrm{mm}$. If we want $\phi$ to be less than $10^{-8} \mathrm{rad} /{ }^{\circ} \mathrm{C}, \Delta L$ must be less than $0.8 \mathrm{~mm}$; thus the tile must be carefully leveled during installation.

A further complication arises in designing the leveling screw-baseplate interface. If the baseplate is tapped to receive the screw, the effective length of the screw depends on exactly where the threads and baseplate are actually in contact. This exact point may be anywhere along the length of the threaded hole, and may change unpredictably when the screw is turned. This would be of no consequence if the baseplate were also made of Invar, because the baseplate would then be an extension of the screw, and its top surface would move according to Eq. (1). However, if the baseplate is made of a more convenient metal such as aluminum, the top of the plate will move more than predicted by Eq. (1), depending on just where the thread makes contact. We have solved this problem by using Invar "nuts" attached to the top of the baseplate, which effectively define the length of the screws regardless of where the threads are engaged within the nuts.

A further potential problem arises from the mechanical interface between the screws and the tile. Most active volcanoes generate earthquakes, and it is therefore desirable to constrain the tiltmeter horizontally. To accomplish this, we place conical holes in small Invar cups which are epoxy bonded to the ceramic tile with the very minimum thickness of epoxy.

Because the thermal coefficient of the tile is different than that of the baseplate, the spacing betwen screws differs from the spacing between cups when the temperature changes. This could force the ends of the screws to move up the sides of the conical holes, thereby inducing large artificial tilts. To prevent this we made the screws long and thin, and the instrument heavy enough, that the screws bend rather than climb out of the conical holes. Since all three screws are of similar size and length, they bend about the same amount and the meter stays very nearly level. To further minimize this effect, we make the baseplate of aluminum which nearly matches the tile in thermal coefficient of expansion $\left(\sim 20 \mathrm{ppm} /{ }^{\circ} \mathrm{C}\right.$ for aluminum vs $\sim 15 \mathrm{ppm} /{ }^{\circ} \mathrm{C}$ for tile).

The bubbles are spring loaded into $V$ grooves in aluminum blocks which are attached to the baseplate. Since thermal gradients are especially serious in these blocks, they are isolated from the baseplate with spring-tensioned nylon screws and identical glass spacers. The blocks are surrounded with insulation and enclosed in a metal box, which produces a thermal time constant for the blocks/bubbles of about an hour and effectively suppresses thermal gradients.

\section{CALIBRATION AND TESTS}

The tiltmeters are calibrated on a simple mechanical tilt table which uses a very rigid optical bench beam $2 \mathrm{~m}$ long. One end of the beam is supported by a cross beam mounted on two large balls which rest on a steel plate. The plate is bonded to a concrete slab floor cast onto the ground. The meter is leveled with its own screws on a table centered on the cross beam. The other end of the long beam is raised or lowered by a differential micrometer screw and monitored by a precision dial gauge. It is possible to reproduce tilts as small as $10 \mu \mathrm{rad}$ with this simple device.

We have conducted three tests of our tiltmeter's performance under relatively controlled conditions. The first took place on a massive isolated concrete pier at the bottom of a 25-m-deep solar telescope pit at Caltech. A Kinemetrics TM-1 tiltmeter ${ }^{5}$ using a Rockwell sensor was operated next to our tiltmeter for several days. By tilting the pier about 50 $\mu \mathrm{rad}$, using a lever against the surrounding floor, we confirmed that our tilt table calibration agreed with the Kinemetrics calibration. The meters tracked each other at the submicroradian level as the pier moved in response to diurnal solar heating of the surrounding building. There were no obvious irregularities in either meter's performance.

A second test was conducted in the Uwekahuna Vault near the Hawaiian Volcano Observatory on Kilauea volcano. Output from our meter was compared with data from a 3-m base line water tube tiltmeter and an Ideal-Arrowsmith ${ }^{13}$ mercury tube tiltmeter. The latter two instruments have operated reliably for many years at Uwekahuna. All three tiltmeters agreed at the $\mu$ rad level during several weeks of simultanous operation.

A third test has been run sporadically since December 1980 at the Dalton Geophysical Observatory tunnel in Southern California. The Fredericks sensors are being compared with a Rockwell sensor using the mechanical and electronic equipment developed for the Rockwell sensor at Caltech. The meters are mounted on the same pier and their outputs are locally recorded. Figure 4 shows a typical comparison. A signal of about $10^{-7}$ rad peak-to-peak amplitude is clearly recorded by both meters. Detailed comparison suggests a mutual uncertainty of less than $2 \times 10^{-8}$ radians rms over most of the record, with some evidence on hysteresis in 


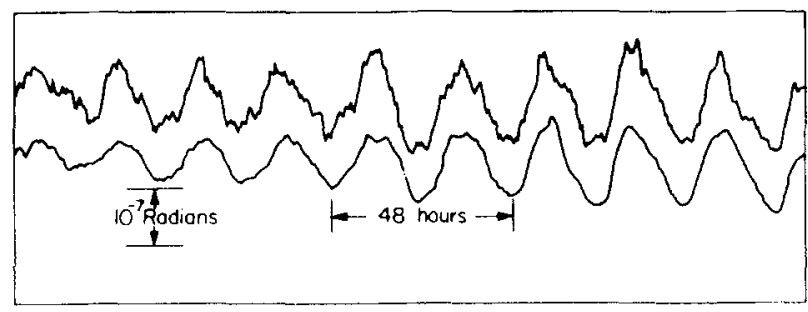

Fig. 4. Comparison of earth tide investigation records from the Fredericks (upper) and Rockwell (lower) bubble tilt sensors.

the Rockwell sensor.

In each of these tests the thermal environment has been benign. Unfortunately, at scientifically interesting sites like Mount St. Helens, tiltmeters must often contend with a severe thermal regime. Several tests have been attempted to separate thermal effects due to our meter's mechanical design from those intrinsic to the Fredericks sensor. None of these tests is entirely satisfactory, but it is clear that at electrical null the sensor temperature coefficient is less than 1 $\mu \mathrm{rad} /{ }^{\circ} \mathrm{C}$. It has not been possible to test our meter's longterm stability except in comparison to the Rockwell sensor at Dalton tunnel; their mutual drift over periods of months is less than $2 \times 10^{-8} \mu \mathrm{rad} /$ day.

\section{INSTALLATION AND OPERATIONAL CONSIDERATIONS}

Since interesting field sites are rarely benign, special precautions must be taken in tiltmeter design to prevent erroneous results. Corrosion protection for certain components is critical, especially at volcanoes where the environment is not only wet but also bathed in $\mathrm{SO}_{2}, \mathrm{H}_{2} \mathrm{~S}, \mathrm{CO}_{2}$, $\mathrm{H}_{2} \mathrm{SO}_{4}, \mathrm{HCl}, \mathrm{HF}$, and other noxious liquids and gases. Liberal use of hard chromium plating, epoxy paints, hard anodize, and silicone grease is essential. Particularly critical are the screw threads, the interfaces between the ends of the screws and the cups, the interface between the glass spacers and the blocks and baseplate, and the surfaces between the blocks. In these places we use very thin layers of silicone grease to protect the contacts (first smear it on, and then wipe it "all" off).

In the field, we protect the entire tiltmeter with a foam cooler, garbage can, metal box, concrete pipe, etc., depending on the site. In each case we try to minimize the effects of the external environment. A discussion of some of our experiences and results at Mount St. Helens is being published elsewhere. $^{2}$
The Fredericks bubbles have an intrinsic thermal coefficient of sensitivity (in contrast to null position) due to the thermal coefficient of electrical conductivity of the electrolyte. This amounts to about $0.4 \% /{ }^{\circ} \mathrm{C}$. Near electrical null this is unimportant for reasonable temperature changes $\left(\sim 10^{\circ} \mathrm{C}\right)$, but in large tilt $(>100 \mu \mathrm{rad})$ situations it requires temperature corrections to the data if a dynamic range greater than $100 \mu \mathrm{rad}$ is required. It also means that in small tilt situations, it is important to operate the sensor near electrical null.

Fortunately in Hawaii, it has been possible to install our meters inside lava tubes, which are nearly isothermal and well coupled to their surroundings. At Mount St. Helens, however, surface temperature changes and poor coupling are both serious problems. Monitoring the baseplate temperature has allowed post-facto correction for thermal effects to about the $10 \mu \mathrm{rad}$ level, completely adequate for monitoring the very large $(>1000 \mu \mathrm{rad})$ tilts observed during recent eruptions. ${ }^{2}$

\section{ACKNOWLEDGMENTS}

We thank James Gunn, Victor Nenow, and DeVere Smith for aid in the design and construction of these meters. We also appreciate the aid and encouragement of the Hawaiian Volcano Observatory staff, particularly Norman Banks and Arnold Okamura. This work was supported by internal Caltech funds, the U.S. Geological survey Volcanic Hazards Program, and USGS Contract 14-08-0001-19753.

a) Seismological Laboratory

${ }^{1}$ R. Decker and B. Decker, Volcanoes (Freeman, San Francisco, 1981)

${ }^{2}$ D. Dzurisin, J. A. Westphal, and D. J. Johnson, Science (submitted 1982). ${ }^{3}$ P. W. Lipman and D. R. Mullineaux, U.S. Geological Survey Professional Paper 1250 (1981), p. 93.

${ }^{4} \mathrm{G}$. L. Cooper, Autonetics Technical Report for Guidance, Control and Flight Mechanics Conference of AIAA (Autonetics, Anaheim, CA, 1970). ${ }^{5}$ Kinemetrics, Inc. 222 Vista Avenue, Pasadena, California 91107

${ }^{6}$ M. J. S. Johnson, U.S. Geological Survey Open File Report (Menlo Park, California, 1976), Report No. 76-556.

${ }^{7}$ C. E. Mortensen and M. J. S. Johnson, J. Geophys. Res. 81, 3561 (1976).

${ }^{8}$ F. Wyatt and J. Berger, J. Geophys. Res. 85, 4351 (1980).

${ }^{9}$ W. T. Kinoshita, D. A. Swanson, and D. B. Jackson, in Physical Volcanology, edited by L. Civetta et al. (Elsevier, Amsterdam, 1974), p. 87

${ }^{10}$ Model 7614, The Fredericks Co., P.O. Box 67, Huntington Valley, Pennsylvania 19006 .

"Any use of trade names is for descriptive purposes only and does not imply endorsement by the USGS.

${ }^{12}$ W. F. Miller, R. J. Geller, and S. Stein, Geophys. J. R. Astron. Soc. 54, 661 (1978).

${ }^{13}$ No longer commercially available to our knowledge. 